\title{
La protección de datos personales: análisis terminológico y jurídico desde presupuestos traductológicos (alemán-español)
}

\author{
Personal data protection: Terminological and legal analysis \\ from translation premises (German-Spanish)
}

\author{
María del Carmen Balbuena Torezano
}

Unversidad de Córdoba. mcbalbuena@uco.es

Recibido: 01.05.2019. Aceptado: 08.07.2019

Resumen: El Reglamento (EU) 2016/679 de Protección de Datos, que deroga la directiva 95/46/EC o Reglamento General de Protección de Datos (RGPD), afecta al tratamiento de los datos personales de los seres humanos, y a la libre circulación de estos datos en la Unión Europea y es de transposición directa; constituye, pues, una norma jurídica esencial en el marco de los derechos individuales de las personas en la era digital. Su aplicación, que entró en vigor en mayo de 2018, ha supuesto la adaptación legislativa y tecnológica de los Estados miembros de la UE y la aplicación de sistemas de control y seguridad que, si bien ya se encontraban en otras disposiciones comunitarias y estatales anteriores, ahora cobran una nueva relevancia, y en algunos casos cambian de denominación. Todo ello supone una amalgama de conceptos nuevos que han de ser comprendidos para dar cumplimiento a esta nueva normativa de forma correcta. Son objetivos de este trabajo: 1) Analizar la terminología que emana del Reglamento (UE) 2016/679 desde dos perspectivas diferentes: por un lado, analizaremos los conceptos jurídicos; por otro, contemplaremos los procedimientos de formación de palabras en lengua alemana; 2) Establecer las equivalencias lingüísticas relativas a los términos estudiados en el par de lenguas alemán-español. Con ello, esperamos obtener un glosario terminológico que resulte de utilidad para la traducción de textos en materia de protección de datos.

Palabras clave: protección de datos personales; traducción jurídica; Reglamento General de Protección de Datos (RGPD); Agencia Española de Protección de Datos (AEPD); terminología (DE-ES).

Abstract: The Regulation (EU) 2016/679, which repeals Directive 95/46/EC or General Data Protection Regulation (GDPR), has an impact on the processing of personal

I) Balbuena Torezano, María del Carmen. 2019. "La protección de datos personales: análisis terminológico y jurídico desde presupuestos traductológicos (alemánespañol)". Quaderns de Filologia: Estudis Lingüistics XXIV: 247-265. doi: 10.7203/QF.24.16309 
data, on the free movement of such data and is an essential law to strengthen individuals' right in the digital age. This Regulation, applied since May 2018, supposes a legislative and technological adaption in the EU States, and the application of control and security systems in these countries, although there were previous Community provisions and state laws. But just now it acquires a new importance and in some cases a change of denomination is necessary. All that implies a vast array of new concepts, which must be understood in order to comply this Regulation. The main purposes of this work are: 1) to analyse the terminology of the Regulation (EU) 2016/679 from two points of view: first of all, we will analyse the legal concepts; secondly, we will study which methods or mechanisms of words composition are used in German; 2) to establish linguistic equivalences of those concepts for the combination German-Spanish. As a result, we hope to achieve a glossary of terms, which will be useful for the translation of texts about personal data protection.

Keywords: personal data protection; legal translation; General Data Protection Regulation (GDPR); Spanish Data Protection Agency (SDPA); terminology (DE-ES). 


\section{Introducción}

La traducción jurídica es una actividad translativa altamente cualificada, que exige un profundo conocimiento de la terminología en las LO y LM, y la correcta comprensión de los conceptos jurídicos. En el caso de textos emanados de procesos de legislación, suele suceder que aparezcan nuevos términos, hasta el momento desconocidos, o bien otros que guardan alguna relación con conceptos y significados contenidos en normativas y textos legales anteriores, modificados, ampliados o derogados por la nueva normativa vigente.

Ante la creación de nuevos términos y conceptos, el traductor deberá, en primer lugar, entender el sentido jurídico de la norma, así como las designaciones y calificaciones que esta contiene; además, habrá de poseer el conocimiento léxico que le permita encontrar equivalencias en la LM. En este sentido, no siembre existen los recursos terminológicos apropiados para el establecimiento de dichas equivalencias, por lo que es necesario confiar en las competencias lingüísticas propias. Partiendo de esta realidad, y tomando como LO, el alemán, es posible establecer la siguiente hipótesis: el conocimiento del concepto jurídico, unido al del funcionamiento de los procedimientos de formación de palabras de la LO, ayudará al traductor a encontrar las equivalencias adecuadas para la traducción de los textos.

En este trabajo presentaremos los términos más relevantes referentes a la protección de datos de carácter personal ${ }^{1}$ desde dos puntos de vista: por un lado, estableceremos los conceptos jurídicos a los cuales hacen referencia los términos seleccionados; por otro, analizaremos los mecanismos empleados en lengua alemana para formar los significantes correspondientes a cada concepto jurídico; todo ello nos llevará al establecimiento de equivalencias lingüísticas en el par de lenguas alemán-español y a la confección de un glosario bilingüe que sea útil para la traducción de textos relativos a la PD, teniendo en cuenta las premisas expuestas en el párrafo anterior.

Iniciaremos el estudio con la presentación de las normativas nacionales y europeas relativas a la PD, para después establecer los fundamentos morfosintácticos y léxico-semánticos que han determinado la extracción de los candidatos a términos; especificaremos la fuente

\footnotetext{
${ }^{1}$ En adelante: PD.
} 
textual que ha servido de base para y los criterios empleados para llevar a cabo la selección léxica. El grueso del estudio lo constituye el análisis de términos desde el punto de vista morfosintáctico, con especial atención a los mecanismos de formación de palabras en lengua alemana (Wortbildung). A tenor de los resultados obtenidos, estableceremos las oportunas conclusiones.

\section{La normativa sobre la protección de datos de carácter personal}

En lo relativo a los datos personales ${ }^{2}$, la protección de las personas físicas es un derecho fundamental, recogido en la Carta de los Derechos Fundamentales de la Unión Europea y el Tratado de Funcionamiento de la Unión Europea.

\subsection{Normativas europeas}

La primera norma que regula la PD en Europa es la Directiva 95/46/ CE del Parlamento Europeo del Consejo, de 24 de octubre de 1995, relativa a la protección de las personas físicas en lo que respecta al tratamiento de datos personales y a la libre circulación de esos datos, también conocida como Reglamento General de Protección de Datos (RGPD). La principal novedad que introducía el RGPD es su aplicación tanto al tratamiento manual como al automatizado de los DaP, si estos figuran en un fichero o están destinados a ser incluidos en él. Esta norma queda derogada por el Reglamento (UE) 2016/679 del Parlamento Europeo y del Consejo de 27 de abril de 2016, que establece que ha de aplicarse únicamente a personas físicas, para las que "debe quedar totalmente claro que se están recogiendo, utilizando, consultando o tratando de otra manera datos personales que le conciernen, así como la medida en que dichos datos son o serán tratados" (2016: 7).

\subsection{Normativas nacionales}

La primera normativa sobre PD se inicia en 1970 en Hessen, Alemania. En el caso de España, esta cuestión sería tratada casi una treintena de años más tarde, con la entrada en vigor de la Ley Orgánica 15/1999 de

\footnotetext{
${ }^{2}$ En adelante: DaP.
} 
13 de diciembre, de Protección de Datos de Carácter Personal ${ }^{3}$, que en su artículo 1 establece que tiene por objeto

(...) garantizar y proteger, en lo que concierne al tratamiento de datos personales, las libertades públicas y los derechos fundamentales de las personas físicas, y especialmente su honor e intimidad personal y familiar (1999: 43088).

Esta ley, referida únicamente a los DaP registrados en soporte físico, era de aplicación en el sector público o privado a) cuando el tratamiento de esos datos se llevara a cabo en territorio español, en el marco de las actividades de un establecimiento del responsable del tratamiento; b) cuando al responsable del tratamiento aun no encontrándose en territorio español, le fuera de aplicación la legislación española en esta materia; c) cuando el responsable del tratamiento de los datos no estuviera en territorio de la UE, pero empleara medios del territorio español para dicho tratamiento, exceptuando los casos en los que dichos medios se emplearan únicamente con fines de tránsito. Por otra parte, la Ley Orgánica es bastante escueta cuando define qué es un dato de carácter personal: “(..$)$ cualquier información concerniente a personas físicas identificadas o identificables" (1999: 43088).

Esta norma fue derogada con efectos de 7 de diciembre de 2018, sin perjuicio de lo previsto en las disposiciones adicional 14 y transitoria 4 de la Ley Orgánica 3/2018, de 5 de diciembre, según establece su disposición derogatoria única 1, que desarrolla lo expuesto en el Reglamento, adaptándolo a la realidad del Estado español, y explicitando de forma detallada aspectos como la PD de las personas fallecidas, o el consentimiento de los menores de edad. Dado que el Reglamento era de obligado cumplimiento desde el 25 de mayo de 2018, esto es, de aplicación directa en los distintos Estados miembros de la UE, y en nuestro país aún no había entrado en vigor la Ley Orgánica 3/2018, que se hallaba en tramitación, el Real Decreto-Ley 5/2018, de 27 de julio, establecía las medidas urgentes para la adaptación del Derecho español a la normativa de la Unión Europea en materia de PD.

La exposición de las normativas vigentes en los sistemas jurídicos alemán, español y europeo pone de manifiesto que ya sea a nivel

\footnotetext{
${ }_{3}$ Disponible en: https://www.boe.es/buscar/pdf/1999/BOE-A-1999-23750-consolidado.pdf. [ Acceso 27/04/2019].
} 
europeo o nacional, la PD guarda una relación directa con la transparencia (Tinnefeld et alii, 2018: 10):

Im europäischen Datenschutz ist der Schutz der Privatheit mit der Forderung nach Transparenz verbunden. Der Betroffene muss klar deutlich darüber informiert werden, welche Daten (Gesundheits- und Sozialdaten, Konto-, Kredit-, und Steuerdaten usw.) über ihn gesammelt werden und zu welchem Zweck, und für wen sie transparent sind ${ }^{4}$.

\section{Algunas cuestiones previas: texto fuente y selección de términos}

Para llevar a cabo el presente hemos tomado como texto fuente el texto alemán del Reglamento (EU) 2016/679, que consta de 173 considerandos previos y 99 artículos, agrupados en 11 capítulos 5 . Los cuatro primeros capítulos contienen la mayor densidad léxica y jurídica, de ahí que sean precisamente estos capítulos los elegidos para la extracción de candidatos a términos, y más específicamente los artículos 1 a 43. Los criterios para la selección de términos están acorde con los objetivos de este trabajo: por un lado, se ha considerado la complejidad conceptual que contiene cada candidato a término desde el punto de vista jurídico, lo que le confiere carácter de unidad léxica especializada (ULE); por otro, se han seleccionado una serie de términos simples (Einwortbennennung) y complejos (Mehrwortbennennung), siguiendo el modelo contemplado por Olmo y López-Mateo (2017: 20), a partir de las definiciones del Instituto Alemán de Normalización (DIN) ${ }^{6}$. Para di-

${ }^{4}$ [La protección de datos en el ámbito europeo es la protección de la privacidad con una clara vinculación a la transparencia. El interesado debe ser claramente informado sobre qué datos suyos son recogidos (salud, datos sociales, de cuentas corrientes, crédito, impuestos, etc.) y para qué fin, y para quién van a ser transparentes estos datos]. Traducción de la autora.

${ }^{5}$ Cfr. https://eur-lex.europa.eu/legal-content/ES/ALL/?uri=CELEX\%3A32016R0679. [Acceso 27/04/2019].

${ }^{6}$ Según el DIN un término simple (Einwortbennenung) es "Eine aus einem Wort bestehende Bennenung. Anmerkung: $\mathrm{Zu}$ den Einwortbennenungen zählen auch die zusammengesetzten einschließlich mit Bindestrich durchgekoppelten Bennenungen (...)" (DIN 2330, 1993, 2.4). ["Denominación que consta de una palabra. Nota: también se consideran términos simples aquellos términos compuestos por términos simples unidos por un guión". Traducción de la autora]. El término compuesto (Mehrwortbennennung) y la agrupación de palabras (Wortgruppe) son "Eine Bennennung, die aus 
cha selección se ha tenido en cuenta la frecuencia de aparición, tomando como candidatos a términos aquellos cuya frecuencia es superior a cinco, y que resultan, desde el punto de vista jurídico y lingüístico, imprescindibles para el desarrollo y la comprensión de la norma tomada como texto fuente.

\section{Principales conceptos relativos a la protección de datos de carácter personal según el Reglamento EU 2016/679}

Para traducir textos relacionados con la PD resulta imprescindible conocer los conceptos jurídicos sobre los que esta se sustenta, para poder determinar la relación conceptual entre ellos, al tiempo que asociar cada uno de estos conceptos con un significante determinado. A continuación, señalamos los conceptos más relevantes en torno a la PD, siguiendo la directiva europea, que ha generado la actual normativa vigente en cada país Estado de la UE:

\subsection{Datos personales (personenbezogene Daten)}

El Reglamento (EU) 2016/679 considera DaP:

Toda información sobre una persona física identificada o identificable (...); se considerará persona física identificable toda persona cuya entidad pueda determinarse, directa o indirectamente, en particular mediante un identificador, como por ejemplo un nombre, un número de identificación, datos de localización, un identificador en línea o uno o varios elementos propios de la identidad física, fisiológica, genética, psíquica, económica, cultural o social de dicha persona (2016: 33).

Los DaP pueden ser identificativos (nombre, apellidos, número del DNI), referidos a la situación laboral o financiera, u otros de carácter similar. Junto a estos hay categorías especiales de datos, entre los que se encuentran: a) datos de salud (Gesundheitsdaten); b) datos de origen étnico o racial (ethnische oder rassische Herkunft); c) datos sobre opiniones políticas (polistische Meinungen); d) datos sobre convicciones

mindestens zwei durch Leerstellen getrenten Wörtern besteht” (DIN 2330, 1993: 2.5). ["Denominación formada por al menos dos palabras separadas entre sí por espacios". Traducción de la autora]. 
religiosas (religiöse Überzeugungen); e) datos sobre afiliaciones sindicales (Gewerkschaftszugehörigkeit); f) datos genéticos (genetische Daten); g) datos biométrios (biometrische Daten); y h) datos sobre orientación y vida sexual (Daten zum Sexualleben und der sexuellen Orientierung).

La lengua alemana es más explícita que la española al hacer referencia a este concepto, pues mientras en español empleamos simplemente el adjetivo "personal", en alemán se emplea la expresión adjetival "que hace referencia/ relativa a las personas" (personenbezogene). Los DaP se refieren siempre a personas físicas (en alemán natürliche Personen, personas "naturales"), y nunca a personas jurídicas (juristische Personen).

\subsection{Tratamiento (Verarbeitung) y limitación del tratamiento (Beschränkung der Verarbeitung) ${ }^{7}$}

El tratamiento es cualquier operación o conjunto de operaciones que se realicen sobre los DaP, ya sea por procedimientos automatizados o no ${ }^{8}$. Estos DaP, no obstante, no pueden ser empleados en el futuro para otro fin distinto a aquel para el cual han sido recogidos, lo que supone, en efecto, una limitación del tratamiento. El siguiente texto es un ejemplo de información sobre el tratamiento y su limitación:

Die Dauer der Speicherung personenbezogener Daten richtet sich nach den jeweiligen gesetzlichen Aufbewahrungsfristen, etwa aus dem Steuer- oder Handelsrecht. Sind diese Aufbewahrungsfristen abgelaufen, werden die jeweiligen personenbezogenen Daten gelöscht, es sei denn diese personenbezogenen Daten sind weitherhin zur Vertragserfüllung oder -anbahnung erforderlich oder wir besitzen weiterhin ein berechtigtes Interesse an der Speicherung dieser Daten?.

\footnotetext{
${ }^{7}$ Obsérvese que el sentido literal de Verarbeitung es “elaboración, manejo, trabajar con algo".

${ }^{8}$ El artículo 4 del Reglamento (2016: 33) establece que estas operaciones pueden ser “(...) por procedimientos automatizados o no, como la recogida, registro, organización, estructuración, conservación, adaptación o modificación, extracción, consulta, utilización, comunicación por transmisión, difusión o cualquier otra forma de habilitación de acceso, cotejo o interconexión, limitación, supresión o destrucción”.

${ }^{9}$ [El tiempo de almacenamiento de datos personales se rige por lo dispuesto por los plazos legales establecidos, fundamentalmente por el derecho fiscal y mercantil. Una vez transcurrido dichos plazos, los datos personales serán eliminados, salvo si dichos datos son útiles para un contrato posterior o si la empresa tuviera un interés legal legítimo
} 


\subsection{Seudonimización (Pseudonymisierung ${ }^{10}$ )}

Los DaP han de ser tratados de forma que no sea posible identificar a la persona física a la que se refieren sin emplear información adicional. Por ejemplo, si al calificar a un alumno, en lugar de su DNI, o su nombre y apellidos, se emplea el número de identificación de alumnado (NIA), no se podrá relacionar dicho número con la persona en cuestión.

\subsection{Fichero (Dateisystem), responsable del tratamiento (Verantwortlicher) y encargado del tratamiento (Auftragsverarbeiter)}

El fichero es el conjunto estructurado de DaP que han sido recogidos para un determinado fin. Este fin está determinado, precisamente, por el responsable del tratamiento, y dichos datos serán tratados por el encargado del tratamiento, por cuenta del responsable de tratamiento. Así, por ejemplo, en el ámbito universitario, el responsable del tratamiento es la Universidad, representada por la persona que ostenta la Secretaría General, y el encargado de un tratamiento determinado podría ser, por ejemplo, un jefe de un servicio concreto.

\subsection{Consentimiento (Einwilligung)}

El consentimiento del interesado es la manifestación de voluntad de aceptar que sus DaP puedan ser incorporados a un fichero y tratados para un fin determinado. Este consentimiento no puede ser implícito, como lo era con la normativa anterior, sino que ha de ser un consentimiento expreso. Se deberá recabar el consentimiento del interesado para un fin determinado; esto implica que si cambia la finalidad del tratamiento es necesario un nuevo consentimiento del interesado ${ }^{11}$.

para conservarlos]. Traducción de la autora. Fuente: https://zdf-enterprises.de/account/ register [Acceso 10/04/2019].

${ }^{10}$ El término Pseudonymisierung tiene un origen latino, por ello para el lector español es fácilmente identificable.

${ }^{11}$ El artículo 4 del Reglamento establece que el consentimiento del interesado es "toda manifestación de voluntad libre, específica, informada e inequívoca por la que el interesado acepta, ya sea mediante una declaración o una clara acción afirmativa, el tratamiento de datos personales que le conciernen" (2016: 33). 


\subsection{Licitud (Rechtmäßigkeit), lealtad (nach Treu und Glauben) y transparencia (Transparenz)}

Son tres principios rectores de la PD, en virtud de los cuales estos datos han de ser tratados de manera lícita, leal y transparente. Es decir, el tratamiento de los DaP ha de estar amparado en alguna de las bases jurídicas que regula el Reglamento, y antes de ser tratados, el interesado ha debido recibir la información necesaria sobre el objeto y los fines del tratamiento, sus consecuencias y los posibles riesgos.

\subsection{Responsabilidad proactiva (Rechenschaftspflicht ${ }^{12}$ )}

Cuando hablamos de responsabilidad proactiva hacemos referencia a que los responsables y encargados de los tratamientos no solo han de cumplir con los principios que establece el Reglamento, sino que han de estar en disposición de poder demostrar dicho cumplimiento.

\subsection{Minimización de datos (Datenminimierung)}

Principio por el cual se tomarán únicamente aquellos DaP necesarios, pertinentes y adecuados al tratamiento que ha de realizarse. No es posible recabar datos adicionales para ampliar información.

\subsection{Exactitud (Richtigkeit)}

Principio en virtud del cual los datos recogidos han de ser exactos y actualizados.

\subsection{Integridad y confidencialidad (Integrität und Vertraulichkeit)}

Principio por el cual el tratamiento de los datos ha de garantizar la seguridad necesaria para impedir un manejo ilícito o la pérdida de los datos.

\footnotetext{
${ }^{12}$ El primer término de esta composición (Rechenschaft) tiene el significado literal de "cuentas", en el mismo sentido que la expresión jemandem für etwas Rechenschaft ablegen ("rendir cuentas a alguien por algo"). Junto a -pflicht ("deber, obligación"), el término alemán recoge el concepto de "obligación del responsable de los DaP de garantizar su seguridad, y de poder demostrar que cumple con los principios establecidos en el Reglamento".
} 


\subsection{Ejercicio de los derechos del interesado (Ausübung der Rechte der betroffenen Person)}

El Reglamento concede al interesado una serie de derechos relacionados con sus DaP:

- Derecho de acceso (Auskunftsrecht). El interesado tendrá derecho a obtener del responsable del tratamiento confirmación de si se están tratando o no DaP que le conciernen, así como información relativa a distintos aspectos de estos $\mathrm{DaP}$ y este tratamiento, en virtud de lo dispuesto en el artículo 15 del Reglamento. El responsable de los datos está obligado a facilitar al interesado una copia de los DaP que están siendo tratados. Veamos un ejemplo, tomado del RGPD:

Sie haben das durch die DSGVO gewährte Recht, jederzeit von dem Verantwortlichen unentgeltliche Auskunft über zu Ihrer Person gespeicherten personenbezogenen Daten sowie eine Kopie dieser Auskunft zu erhalten ${ }^{13}$.

- Derecho de rectificación (Recht auf Berichtigung). El interesado tendrá derecho a que sus datos sean rectificados sin dilación indebida, o a completar aquellos que estén incompletos. Como ejemplo tomamos el siguiente fragmento:

Sie haben das Recht, die unverzügliche Berichtigung der Sie betreffenden unrichtigen personenbezogenen Daten zu verlangen. Ferner steht Ihnen das Recht zu, unter Berücksichtigung der Zwecke der Verarbeitung, die Vervollständigung unvollständiger personenbezogener Daten, auch mittels einer ergänzenden Erklärung, zu verlangen ${ }^{14}$.

\footnotetext{
${ }^{13}$ [En virtud de lo dispuesto en el RGPD usted tiene derecho a solicitar al responsable de los datos en todo momento información sobre aquellos datos personales almacenados que le conciernan, así como una copia de dicha información]. Traducción de la autora. Fuente: https://zdf-enterprises.de/account/register. [Acceso 10/04/2019].

${ }^{14}$ [Tiene derecho a exigir la rectificación de aquellos datos personales que le conciernan, así como también, en virtud de la finalidad para la cual fueron recabados dichos datos, completar aquellos que estén incompletos, incluso con una aclaración adicional]. Traducción de la autora. Fuente: https://zdf-enterprises.de/account/register. [Acceso 10/04/2019].
} 
- Derecho de supresión o "derecho al olvido" (Recht auf Löschung oder "Recht auf Vergessenwerden"). El interesado tendrá derecho a que sean suprimidos los $\mathrm{DaP}$ que le conciernan sin dilación indebida. El siguiente fragmento es un ejemplo:

Sie haben das Recht, von dem Verantwortlichen zu verlangen, dass die Sie betreffenden personenbezogenen Daten unverzüglich gelöscht werden, sofern einer der folgenden Gründe zutrifft und soweit die Verarbeitung nicht erforderlich ist:

Wurden die personenbezogenen Daten von uns öffentlich gemacht und ist unser Unternehmen als Verantwortlicher gemäß Art. 17. Abs. 1 DSGVO zur Löschung der personenbezogenen Daten verpflichtet, so treffen wir unter Berücksichtigung der verfügbaren Technologie und der Implementierungskosten angemessene Maßnahmen, auch technischer Art $(\ldots)^{15}$.

- Derecho a la limitación del tratamiento (Recht auf Einschränkung der Verarbeitung). El artículo 18 del Reglamento regula el derecho del interesado a obtener la limitación del tratamiento de sus DaP si concurren determinadas circunstancias.

- Obligación de notificación (Mitteilungspflicht). El responsable del tratamiento está obligado a comunicar cualquier rectificación o supresión de $\mathrm{DaP}$ al interesado, así como la limitación del tratamiento, si se hubiere efectuado, conforme a lo establecido en los artículos 16, 17.1 o 18 del Reglamento.

- Derecho a la portabilidad de los datos (Recht auf Datenübertragbarkeit). El artículo 20 del Reglamento establece que el interesado tendrá derecho a recibir los DaP que le incumban de parte del responsable de tratamiento, y a transmitirlo a otro responsable del tratamiento, si concurren algunas circunstancias.

${ }^{15}$ [Tiene derecho a exigir al responsable de los datos que elimine sus datos personales, siempre y cuando no concurra alguna de las siguientes razones: Habiendo sido publicados los datos personales, y estando nuestra empresa obligada a eliminarlos conforme al artículo 17, apartado 1, del RGPD, se procederá teniendo en cuenta la tecnología disponible y los costes de implantación de las medidas adecuadas, también de tipo técnico (...)]. Traducción de la autora. Fuente: https://zdf-enterprises.de/account/register. [Acceso 10/04/2019]. 
- Derecho de oposición (Widerspruchsrecht). El interesado podrá oponerse en cualquier momento a que los $\mathrm{DaP}$ que le conciernen sean objeto de un tratamiento, aun cuando haya dado su consentimiento explícito para que sus DaP sean tratados. Así lo muestra el siguiente texto:

Sie haben das Recht, aus Gründen, die sich aus ihrer besonderer Situation ergeben, jederzeit gegen die Verarbeitung Sie betreffender personenbezogener Daten, die aufgrund von Art. 6 Abs. 1 Buchstaben e oder f DSGVO erfolgt, Widerspruch einzulegen ${ }^{16}$.

\subsection{Registro de actividades de tratamiento (Verzeichnis von Verarbeitungstätigkeiten)}

Cada responsable de tratamiento ha de llevar un registro de las actividades de tratamiento efectuadas bajo su responsabilidad. El artículo 30 del Reglamento establece la información que debe contener dicho registro.

\subsection{Delegado de protección de datos (Datenschutzbeauftragter)}

La figura del delegado de protección de datos (DPD) se ha convertido en elemento esencial. Es de obligado cumplimiento en el caso de los organismos públicos, como son las universidades, y tiene como principal objetivo asesorar al responsable y al encargado de tratamiento. Es una figura independiente, que supone el punto de contacto entre la autoridad de control y el responsable del tratamiento.

\subsection{Autoridad de control (Aufsichtsbehörde)}

Es la autoridad independiente establecida por un Estado miembro para supervisar la aplicación del Reglamento. En España la autoridad de control es la Agencia Española de Protección de Datos (AEPD). Su homóloga alemana es el Bundesbeauftragter für den Datenschutz und

\footnotetext{
${ }^{16}$ [En virtud de lo dispuesto en el artículo 6, apartado 1, letras e) o f) del RGPD, usted puede ejercer su derecho a oponerse al tratamiento de sus datos personales, de forma razonada y fundamentada]. Traducción de la autora. Fuente: https://zdf-enterprises.de/ account/register. [Acceso 10/04/2019].
} 
die Informationsfreiheit $(\mathrm{BfDI})^{17}$; en Austria, la Republik Österreich Datenschutzbehörde (DSB) ${ }^{18}$; , finalmente, en Suiza está el Eidgenössischer Datenschutz und Öffentlichkeitsbeauftragter (EDÖB) ${ }^{19}$.

3.15 Evaluación del impacto (Datenschutz-Folgenabschätzung), seguridad del tratamiento (Sicherheit der Verarbeitung) y violación de la seguridad de los datos personales (Verletzung des Schutzes personenbezogener Daten)

El responsable del tratamiento ha de estimar una evaluación del impacto de dicho tratamiento, cuando se empleen nuevas tecnologías. Para ello, deberá contar con el asesoramiento del DPD. El artículo 35 del Reglamento establece los supuestos en los que es obligatoria esta evaluación. Por otra parte, el responsable y el encargado del tratamiento han de aplicar aquellas medidas técnicas y organizativas que sean oportunas para asegurar un nivel de seguridad adecuado para cada tratamiento, que garantice su confidencialidad, seudonimización y evaluación. En caso de que se produzca una violación de la seguridad de los DaP, el responsable del tratamiento deberá notificarlo a la autoridad de control sin dilación injustificada, y, a ser posible, en el transcurso de las 72 horas tras haber tenido constancia de ella, a menos que tenga la certeza de que dicha violación no constituirá, casi con total seguridad, un riesgo para los derechos y libertades de las personas físicas. Si sobrepasase ese plazo, deberá justificar la dilación.

\section{Terminología alemán-español relativa a la protección de datos de carácter personal}

Para el análisis morfosintáctico que aquí proponemos hemos seguido lo dispuesto por López-Campos (2004) en su estudio sobre los procesos de formación de palabras (Wortbildungslehre), así como las aportaciones de Erben (1993) y Fleischer y Barz (1995).

\footnotetext{
${ }^{17} \mathrm{Vid}$. https://www.bfdi.bund.de/DE/Home/home_node.html. [Acceso 28/04/2019].

${ }^{18}$ Vid. https://www.dsb.gv.at/. [Acceso 28/04/2019].

${ }^{19} \mathrm{Vid}$. https://www.edoeb.admin.ch/edoeb/de/home.html. [Acceso 28/04/2019].
} 


\subsection{Términos obtenidos por composición}

La mayoría de los términos presentes en la normativa europea en lengua alemana se han formado por composición. Así, por ejemplo, a partir del hiperónimo Recht ("derecho"), nos encontramos con nuevos términos formados por composición determinativa, como los siguientes:

Grund- $\quad+$-recht $>$ Grundrecht (derecho fundamental)

Auskunft- $\quad+$-recht $>$ Auskunftsrecht (derecho de acceso)

Widerspruch + -recht $>$ Widerspruchsrecht (derecho de oposición)

En algunos casos el significado del término de nueva creación no se corresponde completamente con el significado de los términos que lo conforman. Así, Auskunftsrecht ("derecho de acceso"), proviene de los términos Auskunft ("información") y Recht ("derecho"). La equivalencia española, sin embargo, hace referencia a "acceso", entendiéndose que el interesado tiene "acceso a la información". Otros ejemplos de composición determinativa son los siguientes:

\begin{tabular}{|c|c|c|c|}
\hline Mitteilung- & + -pflicht & $>$ & $\begin{array}{l}\text { Mitteilungspflicht } \\
\text { (obligación de notificación) }\end{array}$ \\
\hline Rechenschaft- & + -pflicht & $>$ & $\begin{array}{l}\text { Rechenschatspflicht } \\
\text { (responsabilidad proactiva) }\end{array}$ \\
\hline Aufsicht- & + -behörde & $>$ & $\begin{array}{l}\text { Aufsichtsbehörde } \\
\text { (autoridad de control) }\end{array}$ \\
\hline Gesundheit- & + -daten & $>$ & $\begin{array}{l}\text { Gesundheitsdaten } \\
\text { (datos relativos a la salud) }\end{array}$ \\
\hline Auftrag- & + -verarbeiter & $>$ & $\begin{array}{l}\text { Auftragsverarbeiter } \\
\text { (encargado de tratamiento) }\end{array}$ \\
\hline personen & + -bezogen & $>$ & $\begin{array}{l}\text { personenbezogen [Daten] } \\
\text { ([datos] relativo a personas, per- } \\
\text { sonal) }\end{array}$ \\
\hline Anwendung- & + -bereich & $>$ & $\begin{array}{l}\text { Anwendungsbereich } \\
\text { (ámbito de aplicación) }\end{array}$ \\
\hline
\end{tabular}

Existen también composiciones copulativas, como es el caso de los términos Datenschutz-Folgenabschätzung ("evaluación de impacto relativa a la protección de datos") o Datenschutz-Grundverordnung ("Reglamento General de Protección de Datos"). 


\subsection{Términos obtenidos por derivación}

Igualmente importante es la obtención de términos jurídicos mediante derivación. Son numerosos los sustantivos deverbativos, en su mayoría obtenidos por adición el sufijo -ung, que los convierten en sustantivos mediante un proceso de transposición:

$\begin{array}{lll}\text { einwilligen (v.) } & > & \text { Einwilligung (sust.) } \\ \text { pseudonymisieren (v.) } & > & \text { Pseudonymisierung (sust.) } \\ \text { beschränken (v.) } & > & \text { Beschränkung (sust.) } \\ \text { einschränken (v.) } & > & \text { Einschränkung (sust.) } \\ \text { verarbeiten (v.) } & > & \text { Verarbeitung (sust.) } \\ \text { verletzen (v.) } & > & \text { Verletzung (sust.) }\end{array}$

En otros casos se produce un cambio de categoría gramatical a partir de un adjetivo; dicho cambio queda marcado con el uso de la mayúscula, propia de los sustantivos alemanes:

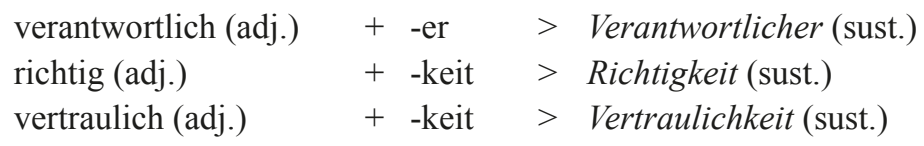

\subsection{Términos obtenidos por composición y derivación}

La complejidad de los conceptos jurídicos se pone de manifiesto en los términos que les corresponden. Por ello, no es infrecuente encontrar denominaciones que son el resultado de procedimientos de formación de palabras igualmente complejos, y reconocer en un mismo vocablo procesos de composición y derivación, de los cuales procede dicho término. Veamos algunos ejemplos:

$$
\begin{aligned}
& \text { Auftrag- }+ \text {-verarbeiten }>\text { - verarbeiter } \Rightarrow \text { Auftragsverarbeiter } \\
& \text { Recht- }+ \text {-mäßig }>\text {-keit } \Rightarrow \text { Rechtsmäßigkeit } \\
& \text { Speicher }+ \text {-begrenz }>\text {-ung } \Rightarrow \text { Speicherbegrenzung }
\end{aligned}
$$

Es paradigmático el término Datenschutzbeauftragter, obtenido por composición determinativa. La primera parte de esta composición ( $D a$ - 
tenschutz-) es, a su vez, un término compuesto; la segunda parte de la composición (-beauftragter) es un término derivado. Composición y derivación dan lugar al término final de siguiente modo:

$$
\begin{array}{lll}
\text { Daten- } & + \text {-schutz } & \Rightarrow \text { Datenschutz } \\
\text { beauftragen (v.) }>\text { beauftragt } & + \text {-er } & \Rightarrow \text { Beauftragter } \\
\text { Datenschutz- } & + \text {-beauftragter } & \Rightarrow \text { Datenschutzbeauftragter }
\end{array}
$$

De estructura similar es el sustantivo Datenschutzerklärung ("información sobre protección de datos"):

$\begin{array}{lll}\text { Daten- } & + \text {-schutz } & \Rightarrow \text { Datenschutz } \\ \text { Erklären (v.) } & + \text {-ung } & \Rightarrow \text { Erklärung } \\ \text { Datenschutz } & + \text {-Erklärung } & \Rightarrow \text { Datenschutzerklärung }\end{array}$

\subsection{Expresiones y frases hechas}

Junto a la terminología que le es propia, el lenguaje jurídico emplea también numerosas expresiones y frases hechas, muchas de ellas incluso procedentes del latín. En el caso de la PD contamos también con expresiones como las siguientes:

Nach Treu und Glauben [sein] $\quad>$ [ser] leal

Verarbeitung nach Treu und Glauben > lealtad

Ausübung der Rechte

$>$ ejercicio de los derechos

Recht auf Berichtigung

Recht auf Löschung

$>$ derecho a rectificación

Recht auf Vergessenwerden

$>$ derecho de supresión

$>$ derecho al olvido

\section{Conclusiones}

Lo expuesto en los epígrafes precedentes nos conduce a las siguientes conclusiones:

- La traducción jurídica aborda múltiples ámbitos de la vida del ciudadano, lo que exige necesariamente una especialización del traductor dentro del ámbito jurídico. 
- En lo relativo a la traducción de textos normativos y otros de carácter jurídico sobre PD, resulta indispensable conocer la legislación vigente, para poder entender los conceptos jurídicos presentes en este tipo de textos.

- Junto a dicho conocimiento jurídico, es preciso el dominio de la LO, de sus mecanismos de creación de nuevos términos y de la formación de palabras, así como de sus estructuras morfosintácticas. Esto es especialmente relevante en el caso del alemán, de gran productividad léxica y por tanto, generadora de numerosos términos especializados.

- El conocimiento de ambos sistemas, el jurídico y el lingüístico, permitirá al traductor establecer las relaciones cognitivas y lingüísticas pertinentes, lo que facilitará, en gran medida, que encuentre las equivalencias adecuadas.

\section{Bibliografía}

AEPD. 2018. Guía del Reglamento General de Protección de Datos para Responsables de Tratamiento. https://www.aepd.es/media/guias/guiargpd-para-responsables-de-tratamiento.pdf. [Acceso 28/04/2019].

AEPD. 2018b. Listado de cumplimiento normativo. https://www.aepd.es/ media/guias/guia-listado-de-cumplimiento-del-rgpd.pdf. [Acceso 28/ 04/2019].

1995. Directiva 95/46/CE del Parlamento Europeo y del Consejo, de 24 de octubre de 1995, relativa a la protección de las personas físicas en lo que respecta al tratamiento de datos personales y a la libre circulación de esos datos. Diario Oficial de las Comunidades Europeas, de 23.11.95, L281-31- L281/50.

Erben, Johannes. 1993. Einführung in die deutsche Wortbildungslehre. Berlín: de Gruyter.

Fleischer, Wolfgang \& Barz, Irmhild. 1995. Wortbildung der deutschen Gegenwartssprache. Tübingen: Niemeyer.

2019. Frankfurter Allgemeine Zeitung. Datenschutzerklärung. https://www. faz.net/datenschutzerklaerung-11228151.html [Acceso: 30/04/2019].

1999. Ley Orgánica 15/1999, de 13 de diciembre, de Protección de Datos de Carácter Personal. Boletín Oficial del Estado. N. ${ }^{\circ} 298,43088-43099$.

2018. Ley Orgánica 3/2018, de 5 de diciembre, de Protección de Datos Personales y garantía de los derechos digitales. Boletín Oficial del Estado. N. ${ }^{\circ} 294,119788-119857$. 
Olmo, Françoise \& López-Mateo, Coral. 2017. Metodología para la extracción de candidatos a términos en el ámbito de la bioquímica. Terminàlia 16: 18-28.

2018. Real Decreto-Ley 5/2018, de 27 de julio, de medidas urgentes para adaptación del Derecho Español a la normativa de la Unión Europea en materia de protección de datos. Boletín Oficial del Estado. N. ${ }^{\circ} 183$, $76249-76257$.

Tinnefeld, Marie Theres et alii. 2018. Einführung in das Datenschutz. Datenschutz und Informationsfreiheit in europäischer Sicht. Berlin/ Boston: Walter de Gruyter.

2016. Verordnung (EU) 2016/679 des Europäischen Parlaments und des Rates von 27. April 2016 zum Schutz natürlicher Personen bei der Verarbeitung personenbezogener Daten, zum freien Datenverkehr und zur Aufhebung der Richtlinie 95/46/EG (Datenschutz-Grundverordnung). Diario Oficial de la Unión Europea de fecha 4.5.2016, L 119/1- L119/88.

Sitios web

AEPD. Agencia Española de Protección de Datos. https://www.aepd.es/. [Acceso 28/4/2019].

DBS. Republik Österreich Datenschutzbehörde. https://www.dsb.gv.at/. [Acceso 28/4/2019].

Der Bundesbeauftragte für den Datenschutz und die Informationsfreiheit. https://www.bfdi.bund.de/DE/Home/home_node.html. [Acceso 28/4/ 2019].

DGD. Deutsche Gesellschaft für Datenschutz. https://dsgvo-musterdatenschutzerklaerung.dg-datenschutz.de/ [Acceso 28/04/2019].

EDÖB. Eidgenössischer Datenschutz und Öffentlichkeitsbeauftragter. https:// www.edoeb.admin.ch/edoeb/de/home.html. [Acceso 28/4/2019]. 